\title{
Transition from stress sensitivity to a depressive state: Iongitudinal twin study
}

Marieke Wichers, Nicole Geschwind, Nele Jacobs, Gunter Kenis, Frenk Peeters, Catherine Derom, Evert Thiery, Philippe Delespaul and Jim van Os

\section{Background}

Daily-life stress sensitivity is associated with depression, but prospective data are lacking.

\section{Aims}

To examine associations between baseline ecological dailylife stress sensitivity and later depression, and to identify genetic and non-genetic factors moderating the transition from stress sensitivity to depression.

\section{Method}

Daily-life stress sensitivity was assessed at baseline in twins $(n=502)$. One baseline and four follow-up measurements of depressive symptoms and negative life events were collected, as well as interview-based diagnoses at baseline and last follow-up. Hypothesised genetic markers were determined.

\section{Results}

Baseline stress sensitivity was associated with increased depressive symptoms at follow-up and risk of major depressive disorder. Both genetic liability and major life events moderated the probability of transition from stress sensitivity to depression.

\section{Conclusions}

Onset of depression is attributable to pre-onset ecological measurements of stress sensitivity, particularly where genetic liability is high and individuals have reached a stage where the influence of competing environmental causes is low.

\section{Declaration of interest}

None.
Recent findings suggest that genetic liability to depression is in part expressed as the tendency to display negative affect in response to minor stressors in the flow of daily life. ${ }^{1}$ Thus, a causal pathway from genetic risk of daily-life stress sensitivity to the development of affective symptoms may be hypothesised. Additional support for a causal pathway from daily-life stress sensitivity to the development of depression can be obtained by assessing the within-individual association between baseline dailylife stress sensitivity and the development of future affective symptoms. We examined this issue in a large general-population female twin sample using experience sampling methodology, ${ }^{1}$ together with prospective dimensional and diagnostic follow-up measurements of depression. In addition, since not everyone with high levels of stress sensitivity will develop depression, factors that might moderate transition to depression were examined. We predicted that indirect measures of genetic risk and polymorphisms in the genes encoding the serotonin transporter (serotonintransporter-linked or 5-HTTLPR) and the gene encoding brainderived neurotrophic factor $(B D N F \text { Val66Met })^{2-5}$ would moderate the transition from experiencing increased daily-life stress sensitivity to future affective symptoms. Furthermore, we hypothesised that the occurrence of negative life events would moderate this transition.

\section{Method}

\section{Sample}

The study sample consisted of 621 women aged 18-46 years from a general-population twin sample from Flanders, Belgium. A total of 218 twin pairs were recruited from the East Flanders Prospective Twin Survey, a population-based survey that has prospectively recorded all multiple births in the province of East Flanders since $1964 .{ }^{6,7}$ Zygosity was determined as described elsewhere. ${ }^{8}$ The project was approved by the local ethics committee and all participants gave written informed consent. The sample was restricted to a single gender, owing to the probability of qualitative differences in the type of environmental stressors that are associated with depression in men and women, ${ }^{9}$ and gender-specific genetic factors for both neuroticism and depression. ${ }^{10}$

\section{Experience sampling method}

The experience sampling method (ESM) is a structured diary technique for assessing individuals in their daily living environment, and has been validated for the use of studying the immediate effects of stressors on mood. ${ }^{1,11-13}$ Participants were given a digital wristwatch and a set of ESM self-assessment forms, the latter collated in a booklet for each day. The wristwatch was programmed to emit a signal ('beep') at an unpredictable moment in each of ten 90 min time blocks between $07.30 \mathrm{~h}$ and $22.30 \mathrm{~h}$ on five consecutive days. At each beep, participants were asked to stop what they were doing and to fill out an ESM self-assessment form, collecting reports of thoughts, current context (activity, persons present and location), appraisals of current situation and mood (for further description see Wichers et al). ${ }^{14}$

\section{Procedure}

The sample was assessed at five time points, including a baseline $\left(T_{0}\right)$ and four follow-up measurements $\left(T_{1}-T_{4}\right)$. The average period between $T_{0}$ and $T_{1}$ was 132 days, between $T_{1}$ and $T_{2}$ it was 91 days, between $T_{2}$ and $T_{3}$ it was 116 days and between $T_{3}$ and $T_{4}$ it was 91 days. The first interview was at the individual's home; follow-up data were collected using questionnaires and telephone interviews. All interviews were performed by trained research psychologists or graduate psychological assistants.

\section{Measurements}

\section{Affective symptoms}

The Structured Clinical Interview for DSM-IV Axis I disorders (SCID-I) was administered in order to obtain current and lifetime diagnosis of major depressive disorder. ${ }^{15}$ The SCID interview was 
conducted at $T_{0}$ and $T_{4}$. At all time points, participants filled in the Symptom Checklist - 90 - Revised (SCL-90-R). ${ }^{16}$ The SCL-90-R depression and anxiety scales were averaged for each measurement occasion, resulting in a combined score reflecting affective symptoms.

\section{Recent negative life events}

At each time point an inventory of recent life events was made, based on the event list of the Interview of Recent Life Events (IRLE) ${ }^{17}$ Participants reported if any of 61 events had occurred in the past 6 months (at baseline) or since the previous time point (at follow-up), and rated the event's impact on a five-point scale ( 1 very pleasant, 5 very unpleasant); for a further description see Jacobs et al. ${ }^{8}$ Congruent with earlier analyses in this sample, ${ }^{8}$ report of an event rated as unpleasant (scoring 4 or 5) defined life event occurrence, and a continuous exposure variable was calculated representing the number of such unpleasant events befalling the individual over the preceding 6 months. Since previous studies have shown that experience of early adversity may potentially confound the association between recent negative life events and risk of depression, ${ }^{18}$ childhood adversity was measured using the shortened version of the 70-item Childhood Trauma Questionnaire (CTQ). ${ }^{19-21}$ At the request of the Twin Registry the most explicit items concerning sexual and physical abuse were omitted; less explicit items were retained.

\section{Daily negative affect and stress appraisals}

Measures of stress and negative affect were collected at each beep in the ESM protocol at $T_{0}$. In order to measure ESM event-related stress (hereafter referred to simply as 'stress'), participants were asked to report the most important event that had happened during the interval between the current and the previous beep. This event was subsequently rated on a seven-point bipolar scale ( -3 very unpleasant, 0 neutral, 3 very pleasant). Responses were recoded to allow high scores to reflect stress ( -3 very pleasant, 0 neutral, 3 very unpleasant). The ESM negative affect score was the mean of six mood adjectives ('I feel insecure/lonely/anxious/ low/guilty/suspicious') rated on seven-point Likert scales.

\section{Genotyping}

Placental tissue for DNA analysis was available for 156 participants, blood samples for 14 and buccal cell samples for 208; the last were obtained using sterile swabs specifically designed for the collection of buccal cell samples for DNA testing (Omni Swabs; Whatman plc, Brentford, UK). Genotyping of 5-HTTLPR was carried out as described previously. ${ }^{8}$ The BDNF Val66Met (rs6265) genotyping was done by KBiosciences (www.kbioscience.co.uk), using an assay based on their in-house KASPar assay. For every monozygotic twin in the sample with genotypic data, the same genotypic data were included for the co-twin, assuming identical genotypes.

\section{Statistical analysis}

Previous studies by our group examined daily-life stress sensitivity using minor stressors as the exposure variable and negative affect in the flow of daily life as the response variable. ${ }^{14,22}$ In this study, however, the response variable was affective symptoms and dailylife stress sensitivity represented the exposure variable, the effect of which on affective symptoms needed to be modelled statistically. Therefore, the variable 'stress sensitivity' was constructed from the ESM observations reflecting the within-individual average effect size of stress on negative affect (based on an average of 27.7 effect sizes per participant). Furthermore, a variable was created representing the average negative life event score measured over $T_{1}-T_{4}$. The variable 'co-twin depression' was created to reflect familial vulnerability to depression and was defined as a past diagnosis of depression in the co-twin. In order to make maximal use of the five measurement occasions of affective symptoms, data were analysed in the long format using multilevel analysis techniques (XTMIXED command in Stata version 10 for Windows). Data from multiple follow-up observations (level 1) were clustered within participants (level 2), who were part of twin pairs (level 3). For the analyses with follow-up DSM-IV diagnosis of depression as the response variable, multilevel logistic regression analyses were conducted using the command XTGEE in Stata version $10 .^{23}$ In these analyses participant observations (level 1) were clustered within twin pairs (level 2).

First, the effect of baseline stress sensitivity on follow-up affective symptoms $\left(T_{1}-T_{4}\right)$ was examined, corrected for affective symptoms at $T_{0}$. Similarly, the association between stress sensitivity and follow-up DSM-IV diagnosis of depression at $T_{4}$ was examined, but here, additionally, all participants with a DSM-IV diagnosis of depression at $T_{0}$ were excluded. In case of significant linear effects, the dose-response relationship of the association was examined by dividing the distribution of stress sensitivity by its tertiles using the XTILE command, thus creating tertile groups. Second, interaction effects were examined between stress sensitivity on one hand, and on the other familial risk (co-twin depression), indirect genetic risk (i.e. examining monozygotic and dizygotic genetic contrasts using the zygosity $\times$ co-twin depression interaction term), direct genetic variation using 5-HTTLPR and BDNF Val66Met genetic polymorphisms, and post-baseline negative life events. These interaction effects were examined for both the dimensional outcome of depression and DSM-IV diagnosis of depression measured at $T_{4}$. All interaction analyses were corrected for SCL-90-R score of affective symptoms at $T_{0}$. In those with a follow-up diagnosis of major depression as the dependent variable, individuals with a diagnosis of DSM-IV depression at baseline were excluded. In the analyses using co-twin measures of familial risk or indirect genetic risk, sensitivity analyses were performed with additional correction for baseline current or past DSM-IV diagnosis of depression. Analyses including negative life events were corrected for the continuous score of early adversity as measured with the shortened version of the CTQ. Variables were standardised in order to report standardised effect sizes.

\section{Results}

The total sample consisted of 621 White women, of whom 610 participated in the ESM procedure. Thirty-one women were excluded because they had missing or too few valid ESM selfreports. Another 49 were excluded owing to missing ESM data and 28 were excluded owing to insufficient ESM data for the construction of the within-individual averaged effect size of stress on negative affect. This resulted in a data-set of 502 individuals who were part of 250 different twin pairs (152 were monozygotic twins, 97 were dizygotic twins and one pair was of unknown zygosity) and included 36 non-twin sisters (see Table 1 for a description of the sample). Data with respect to follow-up affective symptoms were present for 481 participants at $T_{1}, 476$ at $T_{2}, 450$ at $T_{3}$ and $443(88 \%)$ at $T_{4}$. For 391 (78\%) participants, SCID-I diagnoses were available at $T_{4}$. The distribution of genotypes of the polymorphisms of the 5-HTTLPR and BDNF Val66Met was in Hardy-Weinberg equilibrium (BDNF Val/Val 269, Val/Met 112, Met/Met 21, $\chi^{2}=3.8$, d.f. $=1, P>0.05$; 5-HTTLPR long/long 121 , long/short 158 , short/short $76, \chi^{2}=3.2$, d.f. $=1$, $P>0.05)$. Results from analyses regarding the Met/Met variant were not interpreted because too few participants $(n=21)$ were included. 


\begin{tabular}{|c|c|}
\hline Age, years: mean (s.d.) & $27.4(7.4)$ \\
\hline \multicolumn{2}{|l|}{ SCL-90-R score: mean (s.d.) } \\
\hline Baseline & $1.44(0.4)$ \\
\hline Follow-up & $1.35(0.4)$ \\
\hline Number of post-baseline negative life events: mean (s.d.) & $3.8(3.8)$ \\
\hline \multicolumn{2}{|l|}{ Education, \% } \\
\hline College or university degree & 66 \\
\hline Secondary education & 33 \\
\hline Primary education only & 1 \\
\hline \multicolumn{2}{|l|}{ Employment, \% } \\
\hline Employed & 64 \\
\hline Student & 32 \\
\hline Unemployed & 2 \\
\hline Homemaker & 2 \\
\hline Sick leave & 1 \\
\hline SCL-90-R, Symptom Checklist - 90 - Revised. & \\
\hline
\end{tabular}

At baseline, 89 of $502(17.7 \%)$ participants had at some time fulfilled DSM-IV criteria for major depression (hereafter referred to as 'history of major depression') and 51 of 391 (13\%) developed a DSM-IV diagnosis of depression between baseline and $T_{4}$, of whom 14 had a baseline history of major depression. Sixteen per cent of those with high (highest 33\%) daily-life stress sensitivity and with no history of major depression developed major depression in the period between baseline and $T_{4}$, compared with $10 \%$ of those with average sensitivity and only $6 \%$ of those with low sensitivity. Almost half $(46 \%)$ of those who developed major depression had displayed daily-life stress sensitivity at the level of the highest $33 \%$ at baseline. People with a baseline history of major depression showed significantly increased daily-life stress sensitivity $(\beta=0.35, \quad P=0.002)$. A significant association was present between the variables making up the baseline stress sensitivity $\times$ post-baseline negative life events interaction term $(\beta=0.08, P=0.043)$. No other association was present between variables in other interaction terms.

\section{Baseline stress sensitivity and follow-up affective symptoms}

Baseline daily-life stress sensitivity was significantly associated with follow-up affective symptoms $(\beta=0.16, P=0.01$, corrected for baseline). Additionally, a dose-response association was apparent from the comparisons of average $v$. low stress sensitivity $(\beta=0.43, P=0.005)$ and high $v$. low stress sensitivity $(\beta=0.46$, $P=0.003$ ) (Fig. 1(a)). The regression coefficient linear trend was significant $(\beta=0.24, \quad P=0.003)$. Furthermore, baseline stress sensitivity was significantly predictive of major depression at follow-up in participants without current major depression at baseline $(\mathrm{OR}=1.34, P=0.048)$. Again, a dose-response association was apparent from the comparisons of average $v$. low stress sensitivity $(\mathrm{OR}=1.10, P=0.82)$ and high $v$. low stress sensitivity $(\mathrm{OR}=1.75, P=0.16)$; Fig. $1(\mathrm{~b})$. The $\beta$ linear trend, however, was not statistically significant $(\beta=1.34, P=0.14)$. A post hoc analysis showed that excluding additionally those with a past diagnosis of depression at baseline further strengthened the association between stress sensitivity and follow-up diagnosis of depression $(\mathrm{OR}=1.43, P=0.030)$.

\section{Moderation of association between stress sensitivity and later depression}

\section{Familial vulnerability to depression}

There was no interaction between co-twin depression and baseline stress sensitivity in the model of follow-up affective symptoms (a)

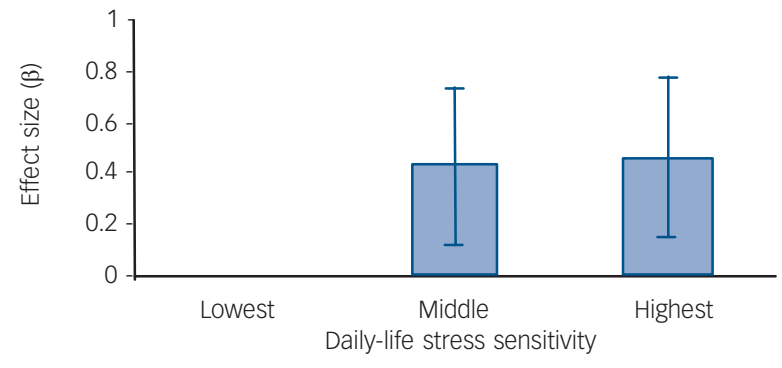

(b)

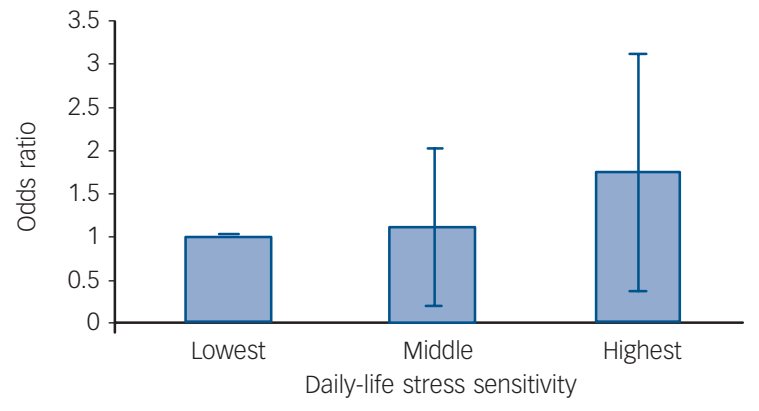

Fig. 1 Dose-response association between daily-life stress sensitivity, divided into tertile groups, and subsequent depression.

(a) Depressive symptoms: standardised effect sizes ( $\beta$ ) for depressive symptoms at follow-up; (b) major depressive disorder: standardised effect in odds ratios (ORs) for a diagnosis of DSM-IV depression at last follow-up assessment. Error bars depict $95 \%$ confidence intervals.

$\left(\chi^{2}=0.88, \quad P=0.35\right)$. However, in probands with co-twin depression the association was greater $(\beta=0.29)$ than in those without co-twin depression $(\beta=0.14)$. A sensitivity analysis, additionally corrected for a baseline history of major depression, showed similar effect sizes (co-twin depression $\beta=0.26$, no co-twin depression $\beta=0.12$; interaction $\beta^{2}=0.74, P=0.39$ ). Similarly, no significant interaction effect was found in the model of followup major depression $\left(\beta^{2}=0.04\right.$, $\left.\mathrm{OR}=0.93, P=0.84\right)$.

\section{Genetic vulnerability to depression}

The three-way interaction effect $\left(\chi^{2}=4.42, P=0.036\right)$ between co-twin depression and baseline stress sensitivity in the model of follow-up affective symptoms was significantly greater within monozygotic (MZ) twin pairs $(\beta=0.039, P=0.047)$ than in dizygotic (DZ) twin pairs $(\beta=-0.024, P=0.30)$. Correction for proband history of DSM-IV depression revealed similar results (interaction $\chi^{2}=4.57, P=0.032$; MZ: $\beta=0.038, P=0.051$; DZ: $\beta=-0.026, P=0.25)$. No significant interaction was found in the model of follow-up major depression $\left(\chi^{2}=2.17\right.$, OR $=0.90$, $P=0.14)$.

\section{Direct genetic markers associated with depression}

The 5-HTTLPR variation significantly moderated the baseline stress sensitivity effect on follow-up affective symptoms (long/ short variant $v$. long/long, $\chi^{2}=4.13, \beta=-0.34, P=0.042$; short/ short variant $v$. long/long, $\chi^{2}=0.02, \beta=0.03, P=0.89$ ). Also, variation in the BDNF Val66Met polymorphism moderated the effect of baseline stress sensitivity on follow-up affective symptoms (Val/Met $v$. Val/Val, $\chi^{2}=4.96, \mathrm{~B}=0.33, P=0.026$ ). No significant moderation by these polymorphisms was found in the model of follow-up major depression. 


\section{Recent negative life events (post-baseline)}

Corrected for baseline depressive symptoms and early adversity, a significant effect of post-baseline negative life events on follow-up depressive symptoms was apparent $(\beta=0.74, P<0.001)$ as well as a significant effect on follow-up major depression $(\mathrm{OR}=1.43$, $P=0.014$ ). In addition, a significant interaction was apparent between post-baseline negative life events and daily-life stress sensitivity in the model of follow-up depressive symptoms $\left(\chi^{2}=5.67, \beta=-0.12, P=0.017\right)$. Although tertile group analyses also showed that the effect of negative life events diminished with increased baseline stress sensitivity, these stratified analyses did not yield significant results (average compared with low stress sensitivity, $\beta=-0.02, P=0.92$; high compared with low stress sensitivity, $\beta=-0.10, P=0.48$; dose-response trend $\beta=-0.06$, $P=0.38$; Fig. 2). There was no significant moderating effect of negative life events in the model of follow-up major depression.

\section{Discussion}

Daily-life stress sensitivity predicted future increase in affective symptoms - shifts to higher positions on the depression continuum, ${ }^{24}$ as well as transition to a full-blown DSM-IV depressive disorder in women without any history of such disorder. In addition, results were compatible with a dose-response relationship: the higher the stress sensitivity, the greater the association with later affective symptoms. However, shifting from low to average stress sensitivity predicted the largest increase in risk, whereas a shift from average to high made a minor extra contribution. The dose-response association did not reach significance for follow-up diagnosis of depression. This is the first study showing associations between stress sensitivity in daily life and future depressive symptoms. Replication is necessary before considering the findings conclusive.

\section{Role of genetic moderators}

The findings (based on both indirect and direct genetic measures) support the hypothesis that genetic factors moderate the effect of daily-life stress sensitivity on a future upward shift along the depression continuum. Indirect genetic vulnerability - sharing $100 \%$ of genes with a person with a history of depression compared with sharing only $50 \%$ - was shown to increase the effect of daily-life stress sensitivity on future depressive symptoms. Also, variations in polymorphisms associated with depression suggest a genetic influence. Participants with a Met allele in the $B D N F$ Val66Met polymorphism, compared with those without

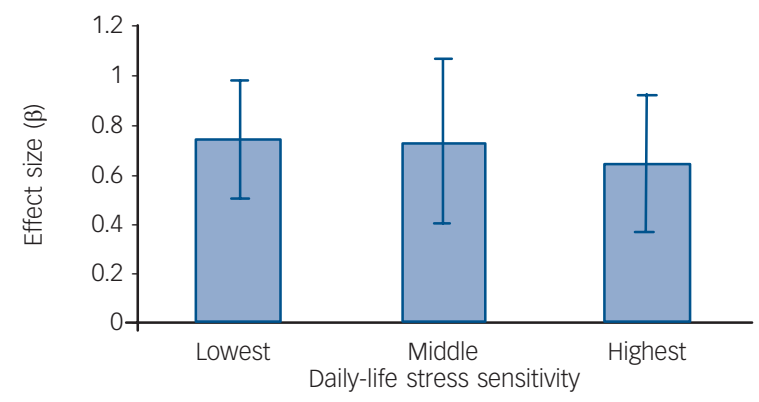

Fig. 2 Post-baseline negative life events interaction with baseline daily-life stress sensitivity in predicting the development of depressive symptoms.

The standardised effect sizes ( $\beta$ ) of negative life events on follow-up depressive symptoms are shown separately for each tertile group of daily-life stress sensitivity. Error bars depict 95\% confidence intervals. this allele, were at increased risk for transition of stress sensitivity to the development of new affective symptoms. The Met allele associated with lower BDNF serum levels than the Val allele has been associated with stress-related outcomes, and metaanalyses (although not all) suggest association between BDNF and depression at various phenotypic levels. ${ }^{25-27}$ In addition, recent studies that examined possible moderation by BDNF Val66Met of the effect of stress on depressive symptoms show a similar increased risk associated with the Met allele. ${ }^{2,28,29}$ Therefore, the current findings agree with existing literature and extend it to minor stressors in the flow of daily life.

In addition, variation in 5-HTTLPR was associated with increased risk of transition to symptomatic affective dysregulation: the short/long variant appeared protective compared with both the short/short and the long/long variants. The literature is inconsistent regarding the effects of 5-HTTLPR in relation to depression: both short/short and long/long variants have been shown to increase risk. ${ }^{5,30,31}$ Therefore, the current finding should be interpreted cautiously and needs replication. To reduce noise and increase the probability of true-positive findings, future research should enrich studies with haplotype analyses or, if possible, data on epigenetic variation conveying information on the transcriptional activity of a particular segment of DNA.

\section{Role of environmental moderators}

Environmental factors also moderate the effect of stress sensitivity. Congruent with previous research, ${ }^{32,33}$ there were main effects of negative life events on both dimensional and dichotomous measures of depression. However, negative life events negatively moderated the effect of stress sensitivity on follow-up affective symptoms. Thus, the higher the level of stress sensitivity the lower the influence of negative life events on future depression. A possible interpretation of this result may be found in findings reported by Kendler et al, ${ }^{34}$ who showed that the influence of life events on depression decreased with multiple past episodes of depression. The authors proposed 'kindling' or 'sensitisation' as the cause of this phenomenon, leading to more autonomous onsets of depression no longer related to major stressful events. Daily-life stress sensitivity has been shown to reflect sensitisation following past stress exposure. ${ }^{18}$ It may be postulated that individuals with an increasing number of past episodes of depression will develop increased daily-life stress sensitivity (which indeed was the case in our sample) and that negative life events now have to 'compete' with daily-life stress sensitivity for new onsets of depression. This would support the theory, as discussed by Monroe \& Harkness, ${ }^{35}$ that stressors do not lose the capacity to trigger depression, but that even small daily-life stressors are able to cause depression, thereby overtaking - in terms of attributable fraction - onsets caused by infrequent major life stressors.

\section{Stress sensitivity as an intermediate phenotype of depression}

There is evidence that ecological daily-life stress sensitivity represents the behavioural expression of genetic risk of depression. ${ }^{14}$ Our study findings indicated that additionally the mechanisms of stress sensitivity mediate onset of depression, i.e. are causal. Factors that substantiate the hypothesis of causality are temporal order, i.e. the exposure preceded the disease outcome; the presence of a dose-response association; biological plausibility; and consistency with other research findings. ${ }^{36}$ Although causality is something that can never be concluded with certainty, the findings presented here support the notion that 
daily-life stress sensitivity causally influences the risk of future depressive symptoms. Furthermore, the strength of the association between the endophenotype daily-life stress sensitivity and depression appears to be dependent on both genetic and environmental factors, illustrating the complexity of the behavioural disorder that is major depression and the need for studies that take into account these complex interacting mechanisms.

\section{Limitations}

There was a certain amount of sample attrition in the follow-up measurements that may have introduced bias. However, the number of people who withdrew from the study was relatively small. Bias due to differential drop-out rates is unlikely since it is not to be expected that people with depression at follow-up with low baseline stress sensitivity dropped out at a different - or higher - rate than those with high baseline stress sensitivity.

The study population was large. There was thus enough power to detect differences in depressive symptoms using a dimensional scale. However, for the analyses using the dichotomous measure 'DSM-IV diagnosis of depression' as the outcome variable, especially for the power-hungry interaction analyses, low power might have contributed to inconclusive findings. Another issue is that exposure to life events is not necessarily a true environmental factor. ${ }^{37}$ It has been shown that genetic factors influence exposure to life events. Therefore, findings should be interpreted accordingly. Finally, measurements of negative life events were based on self-report, as opposed to the use of structured interviews such as the Life Events and Difficulties Schedule, increasing the possibility of retrospective bias and introducing more error in the timing of event occurrence. Although negative life events were not rated specifically with regard to level of contextual threat, the rating of negative emotional attribution in the IRLE arguably constitutes a valid way of identifying those occurrences most likely to shape the risk of future depression.

Marieke Wichers, MSC, PhD, Nicole Geschwind, MSC, Department of Psychiatry and Neuropsychology, South Limburg Mental Health Research and Teaching Network, EURON, Maastricht University, Maastricht, The Netherlands; Nele Jacobs, MSC, PhD, Department of Psychiatry and Neuropsychology, South Limburg Mental Health Research and Teaching Network, EURON, Maastricht University, and Faculty of Psychology, Open University of the Netherlands, Heerlen; Gunter Kenis, MSc, PhD, Department of Psychiatry and Neuropsychology, South Limburg Mental Health Research and Teaching Network, EURON, Maastricht University; Frenk Peeters, MSC, MD, PhD, Department of Psychiatry and Neuropsychology, South Limburg Mental Health Research and Teaching Network, EURON, Maastricht University; Catherine Derom, MSc, PhD, Department of Human Genetics, University Hospital Gasthuisberg Catholic University of Leuven, Belgium; Evert Thiery, MSC, MD, PhD, Association for Scientific Research in Multiple Births, Ghent, Belgium; Philippe Delespaul, MSC, PhD, Department of Psychiatry and Neuropsychology, South Limburg Mental Health Research and Teaching Network, EURON, Maastricht University; Jim van Os, MSC, MD, PhD, MRCPsych, Department of Psychiatry and Neuropsychology, South Limburg Mental Health Research and Teaching Network, EURON, Maastricht University, The Netherlands, and Division of Psychological Medicine, Institute of Psychiatry, London, UK

Correspondence: M. C. Wichers, Department of Psychiatry and Psychology, South Limburg Mental Health Research and Teaching Network, EURON, Vijverdalseweg 1, Concorde Building, Maastricht, The Netherlands. Email: m.wichers@sp.unimaas.n

First received 2 Jul 2008, final revision 11 Aug 2009, accepted 21 Aug 2009

\section{Funding}

This research was supported by The Netherlands Organisation for Scientific Research; the Fund for Scientific Research, Flanders, and Twins, a non-profit association for scientific research in multiple births (Belgium) (to the East Flanders Prospective Survey); and the research in multiple births (Belgium) (to the East Flanders Prospective Survey); and the
EU Framework 6 Integrated Project NewMood (LSHMCT-2004-503474) (to G.K.). M.C.W. was supported by the Dutch Medical Council (VENI grant 916.76.147).

\section{Acknowledgements}

We thank all the twins for their cooperation

\section{References}

1 Csikszentmihalyi M, Larson R. Validity and reliability of the ExperienceSampling Method. J Nerv Ment Dis 1987; 175: 526-36.

2 Kaufman J, Yang BZ, Douglas-Palumberi H, Grasso D, Lipschitz D, Houshyar S, et al. Brain-derived neurotrophic factor-5-HTTLPR gene interactions and environmental modifiers of depression in children. Biol Psychiatry 2006; 59 673-80.

3 Bergstrom A, Jayatissa MN, Mørk A, Wiborg O. Stress sensitivity and resilience in the chronic mild stress rat model of depression; an in situ hybridization study. Brain Res 2008; 1196: 41-52.

4 Caspi A, Sugden K, Moffitt TE, Taylor A, Craig IW, Harrington H, et al. Influence of life stress on depression: moderation by a polymorphism in the 5-HTT gene. Science 2003; 301: 386-9.

5 Hayden EP, Dougherty LR, Maloney B, Olino TM, Sheikh H, Durbin CE, et al. Early-emerging cognitive vulnerability to depression and the serotonin transporter promoter region polymorphism. J Affect Disord 2008; 107 227-30.

6 Loos R, Derom C, Vlietinck R, Derom R. The East Flanders Prospective Twin Survey (Belgium): a population-based register. Twin Res 1998; 1: 167-75.

7 Derom C, Vlietinck R, Thiery E, Leroy F, Fryns JP, Derom R. The East Flanders Prospective Twin Survey (EFPTS). Twin Res 2002; 5: 337-41.

8 Jacobs N, Kenis G, Peeters F, Derom C, Vlietinck R, van Os J. Stress-related negative affectivity and genetically altered serotonin transporter function: evidence of synergism in shaping risk of depression. Arch Gen Psychiatry 2006; 63: 989-96.

9 Kendler KS, Thornton LM, Prescott CA. Gender differences in the rates of exposure to stressful life events and sensitivity to their depressogenic effects. Am J Psychiatry 2001; 158: 587-93.

10 Fanous A, Gardner CO, Prescott CA, Cancro R, Kendler KS. Neuroticism, major depression and gender: a population-based twin study. Psychol Med 2002; 32: 719-28.

11 deVries M, Delespaul P, Dijkman-Caes C. Consequences of depression for the experience of anxiety in daily life. In The Experience of Psychopathology: Investigating Mental Disorders in Their Natural Settings (ed M devries): 141-7. Cambridge University Press, 1992.

12 Delespaul P. Assessing Schizophrenia in Daily Life: The Experience Sampling Method. University of Limburg Press, 1995.

13 Jacobs N, Nicolson NA, Derom C, Delespaul P, van Os J, Myin-Germeys I. Electronic monitoring of salivary cortisol sampling compliance in daily life. Life Sci 2005; 76: 2431-43.

14 Wichers M, Myin-Germeys I, Jacobs N, Peeters F, Kenis G, Derom C, et al. Genetic risk of depression and stress-induced negative affect in daily life. Br J Psychiatry 2007; 191: 218-23.

15 First MB, Spitzer RL, Gibbon M, Williams JBW. Structured Clinical Interview for DSM-IV Axis I Disorders. Biometrics Research Department, New York State Psychiatric Institute, 1995.

16 Derogatis LR, Rickels K, Rock AF. The SCL-90 and the MMPI: a step in the validation of a new self-report scale. Br J Psychiatry 1976; 128: 280-9.

17 Paykel ES. The Interview for Recent Life Events. Psychol Med 1997; 27: 301-10.

18 Wichers M, Schrijvers D, Geschwind N, Jacobs N, Myin-Germeys I, Thiery E, et al. Mechanisms of gene-environment interactions in depression: evidence that genes potentiate multiple sources of adversity. Psychol Med 2009; 39 : 1077-86.

19 Bernstein DP, Ahluvalia T, Pogge D, Handelsman L. Validity of the Childhood Trauma Questionnaire in an adolescent psychiatric population. J Am Acad Child Adolesc Psychiatry 1997; 36: 340-8.

20 Arntz A, Wessel I. Jeugd Trauma Vragenlijst [Dutch version of the Childhood Trauma Questionnaire]. Maastricht: Author, 1996.

21 Bernstein DP, Fink L, Handelsman L, Foote J, Lovejoy M, Wenzel K, et al. Initial reliability and validity of a new retrospective measure of child abuse and neglect. Am J Psychiatry 1994; 151: 1132-6.

22 Myin-Germeys I, van Os J, Schwartz JE, Stone AA, Delespaul PA. Emotional reactivity to daily life stress in psychosis. Arch Gen Psychiatry 2001; 58: 1137-44.

23 Snijders T, Bosker R. Multilevel Analysis: An Introduction to Basic and Advanced Multilevel Modeling. Sage, 1999. 
24 Kendler KS, Gardner CO. Boundaries of major depression: an evaluation of DSM-IV criteria. Am J Psychiatry 1998; 155: 172-7.

25 Verhagen $M$, van der Meij A, van Deurzen PA, Janzing JG, Arias-Vásquez A Buitelaar JK, et al. Meta-analysis of the BDNF Val66Met polymorphism in major depressive disorder: effects of gender and ethnicity. Mol Psychiatry 2008; doi: 10.1038/mp2008.109.

26 Sen S, Duman R, Sanacora G. Serum brain-derived neurotrophic factor, depression, and antidepressant medications: meta-analyses and implications. Biol Psychiatry 2008; 64: 527-32.

27 Brunoni AR, Lopes M, Fregni F. A systematic review and meta-analysis of clinical studies on major depression and BDNF levels: implications for the role of neuroplasticity in depression. Int I Neuropsychopharmacol 2008; 11 1169-80.

28 Aguilera M, Arias B, Wichers M, Barrantes-Vidal N, Moya J, Villa H, et al. Early adversity and 5-HTT/BDNF genes: new evidence of gene-environment interactions on depressive symptoms in a general population. Psychol Med 2009; 39: 1425-32.

29 Gatt JM, Nemeroff CB, Dobson-Stone C, Paul RH, Bryant RA, Schofield PR, et al. Interactions between BDNF Val66Met polymorphism and early life stress predict brain and arousal pathways to syndromal depression and anxiety. Mol Psychiatry 2009; 14: 681-95.

30 Araya R, Hu X, Heron J, Enoch MA, Evans J, Lewis G, et al. Effects of stressful life events, maternal depression and 5-HTTLPR genotype on emotional symptoms in pre-adolescent children. Am J Med Genet B Neuropsychiatr Genet 2009; 150B: 670-82.
31 Laucht M, Treutlein J, Blomeyer D, Buchmann AF, Schmid B, Becker K, et al. Interaction between the 5-HTTLPR serotonin transporter polymorphism and environmental adversity for mood and anxiety psychopathology: evidence from a high-risk community sample of young adults. Int J Neuropsychopharmacol 2009; 12: 737-47.

32 Bouma EM, Ormel J, Verhulst FC, Oldehinkel AJ. Stressful life events and depressive problems in early adolescent boys and girls: the influence of parental depression, temperament and family environment. J Affect Disord 2008; 105: 185-93.

33 Comijs HC, Beekman AT, Smit F, Bremmer M, van Tilburg T, Deeg DJ. Childhood adversity, recent life events and depression in late life. J Affect Disord 2007; 103: 243-6.

34 Kendler KS, Thornton LM, Gardner CO. Stressful life events and previous episodes in the etiology of major depression in women: an evaluation of the 'kindling' hypothesis. Am J Psychiatry 2000; 157: 1243-51.

35 Monroe SM, Harkness KL. Life stress, the 'kindling' hypothesis, and the recurrence of depression: considerations from a life stress perspective. Psychol Rev 2005; 112: 417-45.

36 Bradford-Hill A. The environment and disease: association or causation? Proc $R$ Soc Med 1965; 58: 295-300.

37 Eaves L, Silberg J, Erkanli A. Resolving multiple epigenetic pathways to adolescent depression. J Child Psychol Psychiatry 2003; 44: 1006-14.

\section{Poems \\ by doctors}

\section{Dead Certainty}

\section{Roy Salole}

As a child I was asked what I wanted to be,

'A doctor,' I said without thinking.

I could not perceive

the death beyond my childish horizon.

Now a man, my limits are shrinking

all around, as death closes in.

Through my stethoscope I hear death's rattle

my rubber hammer falls from my hands

in battle; at each skirmish I lose

death raises a cheer;

though I cannot but leer

at his magnificent finality.

'You are a bad loser,' he says, adding

'Why take sides against

dead certainty.'

Roy M. Salole was born in Aden, South Yemen. He studied medicine in London and practises psychiatry in Ottawa. This poem is from The Naked Physician: Poems about the Lives of Patients and Doctors, edited by Ron Charach (Quarry Press). Reprinted by kind permission of the author.

Chosen by Femi Oyebode. 